\title{
CURVAS DE DOSE-RESPOSTA DE BIÓTIPO RESISTENTE DE Brachiaria plantaginea A HERBICIDAS INIBIDORES DA ACCase
}

\author{
Pedro Jacob Christoffoleti ${ }^{1}$
}

'Professor Associado. USP/ESALQ, Departamento de Produção Vegetal. Caixa Postal 09. Piracicaba, SP 13418-900 pjchrist@esalq.usp.br

\begin{abstract}
RESUMO
O desenvolvimento de biótipos de capim-marmelada (Brachiaria plantaginea) resistentes aos herbicidas inibidores da acetil coenzima A carboxilase (ACCase) tem ocorrido com frequência em áreas produtoras de soja, especialmente no Estado do Paraná. No entanto, poucos são os relatos existentes na literatura sobre a comprovação científica desta resistência através de curvas de dose-resposta. O presente trabalho foi realizado com o objetivo de estabelecer curvas de dose-resposta e determinar os $\mathrm{GR}_{50}$ e $\mathrm{GR}_{80}$ de um biótipo de capim-marmelada, com suspeita de resistência a herbicidas inibidores da ACCase. Para isso, foram utilizados sete herbicidas, pertencentes aos grupos químicos ariloxifenoxipropiônicos (haloxyfop-methyl, quizalofop-p-ethyl, fluazifop-p-butil e fenoxaprop-p-ethyl) e ciclohexanodionas (sethoxydim, clethodim e butroxydim), cada um aplicado nas proporções de $0,1,1,0,10$ e 100 vezes a dose recomendada de cada produto. O experimento foi instalado em condições de casa-de-vegetação do Departamento de Produção Vegetal da ESALQ/USP, em vasos plásticos com $500 \mathrm{ml}$ de capacidade. A aplicação dos herbicidas foi feita em pós-emergência da planta daninha, no estádio de 2 a 3 perfilhos. O biótipo de capim-marmelada mostrou resistência cruzada aos dois grupos químicos inibidores da ACCase, porém o grau de resistência foi variável entre os herbicidas.
\end{abstract}

Palavras-chave: ariloxifenoxipropiônicos, ciclohexanodionas, resistência a herbicidas.

\section{ABSTRACT \\ Dose-response curves of a resistant Brachiaria plantaginea biotype to ACCase inhibitor herbicides}

The development of a Brachiaria plantaginea resistant biotype to ACCase inhibitor herbicides has occurred in several soyabean production areas in Brazil, especially in Paraná State. However, little research has been conducted in order to prove scientifically the resistance using dose-response curves. In order to draw dose-response curves and determine $\mathrm{GR}_{50}$ and $\mathrm{GR}_{80}$ of a suspected B. plantaginea biotype, herbicides of the chemical groups ariloxyphenoxypropionic acid (haloxyfop-methyl, quizalofop-p-ethyl, fluazifop-p-butil and fenoxaprop-p-ethyl) and cyclohexanodiones (sethoxydim, clethodim and butroxydim) were sprayed at rates of $0.1,1.0,10$ and 100 times the recommended rate. The experiment was installed in the greenhouse of the Plant Production Department of USP/ESALQ, in $500 \mathrm{ml}$ plastic pots. The herbicides were sprayed at postemergence when the weed was at 2 to 3 shoot stage. The Brachiaria plantaginea biotype studied showed cross resistance to both ACCase inhibitor herbicides; however, the degree of resistance varied among the individual herbicides.

Key words: ariloxyphenoxypropionic acids, cyclohexanodiones, herbicide resistance. 


\section{INTRODUÇÃO}

O uso de herbicidas inibidores da ACCase (ariloxifenoxipropionatos e ciclohexanodionas) na cultura da soja, especialmente nas áreas de plantio direto no Estado do Paraná, tem provocado o aparecimento de biótipos resistentes de capim-marmelada (Vidal \& Fleck, 1997; Gazziero et al., 1997; Christoffoleti et al., 1998; Cortez, 2000).

O capim-marmelada tem sido uma planta daninha de grande importância na cultura da soja, especialmente pelas suas características biológicas, ou seja, ampla distribuição em áreas cultivadas, alta capacidade reprodutiva e de produção de sementes, rápida reposição do banco de sementes, reprodução alógama e plasticidade fenotípica (Seldulsky, 1978). Estas características são favoráveis ao desenvolvimento de biótipos de plantas daninhas resistentes.

Dentre as principais espécies de plantas daninhas que desenvolveram resistência aos herbicidas inibidores da ACCase destacam-se: Alopecurus myosuroides, Avena fatua, Avena sterilis, Digitaria sanguinalis, Eleusine indica, Lolium multiflorum, Lolium rigidum, Setaria viridis, Setaria faberii, Sorghum halepense e Brachiaria plantaginea, sendo esta última no Brasil (DeVine, 1997). Os primeiros casos ocorreram depois de 7-10 anos da introdução dos herbicidas ariloxifenoxipropiônicos e ciclohexanodionas (Heap \& Knight, 1982; Thai et al., 1985).

Os biótipos de plantas daninhas resistentes aos herbicidas inibidores da ACCase apresentam padrões diferentes de resistência. Alopecurus myosuroides apresenta resistência ao diclofop-methyl e ao fenoxaprop-p-ethyl; porém, o nível de resistência a outros ariloxifenopropionatos varia consideravelmente (Preston, 1994). Outros trabalhos na literatura também tem demostrado diferenças nos níveis de resistência de biótipos de espécies gramíneas a inibidores da ACCase, especialmente entre os herbicidas sethoxydim e clethodim, sendo normalmente menos sensível a este último (Sasaki et al., 1995; Heap \& Knight, 1990; Heap et al., 1993).

Com o objetivo de comprovar a resistência de um biótipo de capim-marmelada (Brachiaria plantaginea) aos herbicidas inibidores da ACCase, em culturas de soja da região de Guarapuava, estado do Paraná, foi desenvolvida a presente pesquisa através da construção de curvas de doseresposta e obtenção de valores do $\mathrm{GR}_{50}$ e $\mathrm{GR}_{80}$, aplicando herbicidas inibidores da ACCase, pertencentes aos grupos químicos ariloxifenoxipropiônicos e ciclohexanodionas.

\section{MATERIAL E MÉTODOS}

O experimento foi conduzido na casa de vegetação do Departamento de Produção Vegetal da Escola Superior de Agricultura "Luiz de Queiroz" - Universidade de São Paulo, em Piracicaba, SP. A metodologia descrita a seguir foi inicialmente desenvolvida por Saari et al. (1992), e foi usada neste experimento com algumas modificações.

\section{Material Vegetal}

As sementes de $B$. plantaginea, supostamente resistentes, foram coletadas na região de Guarapuava, Estado do Paraná, em áreas cultivadas com soja, as quais tinham sido pulverizadas há vários anos com herbicidas inibidores da ACCase. Nos dois últimos anos observaram-se escapes de $B$. plantaginea, que normalmente vinham sendo controlados. As aplicações destes herbicidas nas áreas de escape não tinham sido eficientes para controlar esta espécie, por isso, suspeitou-se tratar de um caso de resistência cruzada da planta daninha a estes produtos (ariloxifenoxipropionatos e ciclohexanodionas).

As sementes foram semeadas em copos plásticos de $500 \mathrm{ml}$, contendo substrato à base de solo e material orgânico. Os vasos foram irrigados de acordo com a necessidade para manter a umidade satisfatória e garantir o perfeito crescimento das plantas. Vasos com plantas uniformes e vigorosas foram selecionados para serem tratados com herbicidas quando as plantas estavam com 2 a 3 perfilhos, aproximadamente.

\section{Tratamentos}

Os herbicidas foram aplicados sobre a parte aérea das plantas de capim-marmelada, com um pulverizador estacionário de precisão proporcionando um consumo de calda de 300 l/ha. Aos herbicidas foram adicionados surfactantes conforme a recomendação do fabricante. Após o tratamento com os herbicidas as plantas retornaram à casa-de-vegetação, sendo irrigadas conforme necessidade.

As doses (Tabela 1) foram determinadas de modo a incluir a dose $\mathrm{GR}_{50}$ (dose de herbicida necessária para inibir $50 \%$ do crescimento das plantas daninhas), bem como $\mathrm{GR}_{80}$

\section{Avaliações}

O efeito dos herbicidas no crescimento das plantas foi avaliado aos 21 dias após a aplicação dos tratamentos (DAA), através da percentagem de controle visual, onde $0 \%$ representa nenhum controle e $100 \%$ controle total da planta daninha.

\section{Análise dos resultados}

Para análise dos dados coletados foi ajustado o modelo sigmoidal (logístico), com transformação da variável dependente em logaritmo de base 10. A variável explanatória é a dose, e a variável resposta é a percentagem de controle:

$$
\% \text { de controle }=\frac{\left(A_{1}-A_{2}\right)}{\left(1+\left(\frac{X}{X_{0}}\right) \cdot P\right)+A_{2}}
$$


Bítipo resistente de Brachiaria plantaginea a inibidores da ACCase

Tabela 1. Doses dos herbicidas inibidores da ACCase, aplicados em condições de pós-emergência, no experimento instalado em casa-de-vegetação. USP/ESALQ, Piracicaba, SP. 1997.

\begin{tabular}{|c|c|c|c|c|c|c|}
\hline \multirow[b]{2}{*}{ Herbicida } & \multicolumn{2}{|c|}{ Dose recomendada } & \multicolumn{4}{|c|}{ Dose de p.c. (l/ha) } \\
\hline & i.a. ${ }^{a}(\mathrm{~kg} / \mathrm{ha})$ & p.c. ${ }^{b}(1 / h a)$ & $0,1 \mathrm{D}$ & 1D & $10 \mathrm{D}$ & 100D \\
\hline Setoxydim $^{\mathrm{d}}$ & 0,230 & 1,25 & 0,125 & 1,25 & 12,5 & 125 \\
\hline Clethodim $^{\mathrm{e}}$ & 0,108 & 0,45 & 0,045 & 0,45 & 4,5 & 45 \\
\hline Butroxydim ${ }^{f}$ & 0,525 & $0,25^{\mathrm{c}}$ & $0,025^{\mathrm{c}}$ & $0,25^{\mathrm{c}}$ & $2,5^{\mathrm{c}}$ & $25^{\mathrm{c}}$ \\
\hline Halox yfop-metil $^{\mathrm{g}}$ & 0,060 & 0,50 & 0,050 & 0,50 & 5,0 & 50 \\
\hline Quizalofop-p-ethyl & 0,100 & 2,00 & 0,200 & 2,00 & 20,0 & 200 \\
\hline Fluazifop-p-butil & 0,250 & 2,00 & 0,200 & 2,00 & 20,0 & 200 \\
\hline Fenoxaprop-p-ethyl & 0,120 & 0,50 & 0,050 & 0,50 & 5,0 & 50 \\
\hline
\end{tabular}

${ }^{\mathrm{a}}$ Ingrediente ativo; ${ }^{\mathrm{b}}$ Produto comercial; ${ }^{\mathrm{c}}$ Valor em g/ha; ${ }^{\mathrm{d}}$ Adicionado Assist 0,5\% v/v; ${ }^{\mathrm{e}}$ Adicionado Dytrol 0,5\% v/v; ${ }^{\mathrm{f}}$ Adicionado Nymbus $0,5 \% \mathrm{v} / \mathrm{v} ;{ }^{\mathrm{g}}$ Adicionado Joint $0,5 \% \mathrm{v} / \mathrm{v}$.

Sendo $A_{1}$ e $A_{2}$ menor e maior controle obtido, respectivamente; $\mathrm{X}_{0}$ ponto de inflexão; $\mathrm{P}$ taxa de aumento de controle em função da dose.

\section{RESULTADOS E DISCUSSÃO}

Na Tabela 2 estão apresentados os resultados do $\mathrm{GR}_{50}$ e $\mathrm{GR}_{80}$ para os herbicidas estudados. O capim-marmelada exibiu um maior nível de resistência ao herbicida sethoxydim, pois o $\mathrm{GR}_{50}$ foi de 7,44 e o $\mathrm{GR}_{80}$ foi maior que 100 , ou seja, nem a maior dose estudada do herbicida atingiu valor de $80 \%$ de controle. No entanto, o biótipo de capim marmelada estudado praticamente não exibiu resistência para o herbicida clethodim, pois o $\mathrm{GR}_{50}$ e $\mathrm{GR}_{80}$, foram $<0,1$ e 1,38 respectivamente. Para o herbicida fluazifop-p-butil, o índice de resistência também foi elevado, quase que se equiparando com o sethoxydin, assim como para o herbicida fenoxaprop-p-ethyl. Para os demais herbicidas a resistência do biótipo estudado foi intermediária (Tabela 2).

Os resultados obtidos neste experimento, analisados e ajustados através de modelos não lineares descritos na metodologia encontram-se nas Figuras 1 a 7 . Observou-se que, para a maioria dos herbicidas, as doses de $\mathrm{GR}_{50}$ estavam entre a dose recomendada e dez vezes a mesma (Tabela 2 e Figuras 1 a 7).

Apesar da dose $\mathrm{GR}_{50}$ ser relativamente baixa para alguns herbicidas, não é possível afirmar que estes tenham apresentado um controle satisfatório das plantas. Os gráficos das Figuras 1 a 7 indicam controles inferiores a $80 \%$ nas doses $1 \mathrm{D}$, na escala para avaliações visuais considerada, o que representaria um controle aceitável das populações de plantas daninhas. As doses necessárias para um controle de $80 \%$ foram superiores às doses recomendadas, para a maioria dos herbicidas testados, estando estas, em média, entre a dose normal e dez vezes a mesma. Considerando-se as doses de $\mathrm{GR}_{80}$, os herbicidas com maiores problemas de controle foram o fenoxaprop-p-ethyl, fluazifop-p-butil e o sethoxydim, com as doses de $\mathrm{GR}_{80}$ entre 10 e 100 vezes a dose recomendada, sendo que para o sethoxydim o $\mathrm{GR}_{80}$ não foi obtido, pois este está acima de $100 \mathrm{D}$ (Tabela 2 e Figuras 1 a 7). Para os demais, as doses necessárias para um controle satisfatório foram superiores às recomendadas, excetuando-se o herbicida butroxydim.

Tabela 2. Doses dos herbicidas necessárias para inibir 50\% $\left(\mathrm{GR}_{50}\right)$ e $80 \%\left(\mathrm{GR}_{80}\right)$ da população de $B$. plantaginea. USP/ESALQ, Piracicaba, SP. 1997.

\begin{tabular}{lcc}
\hline \multicolumn{1}{c}{ Herbicida } & $\mathbf{G R}_{\mathbf{5 0}}$ & $\mathbf{G R}_{\mathbf{8 0}}$ \\
\hline Sethoxydim & 7,44 & $>100$ \\
Clethodim & $<0,1$ & 1,38 \\
Butroxydim & 2,06 & 4,77 \\
Haloxyfop-methyl & 1,67 & 2,40 \\
Quizalofop-p-ethyl & $<0,1$ & 2,03 \\
Fluazifop-p-butil & 19,43 & 98,69 \\
Fenoxaprop-p-ethyl & 5,17 & 61,65 \\
\hline
\end{tabular}

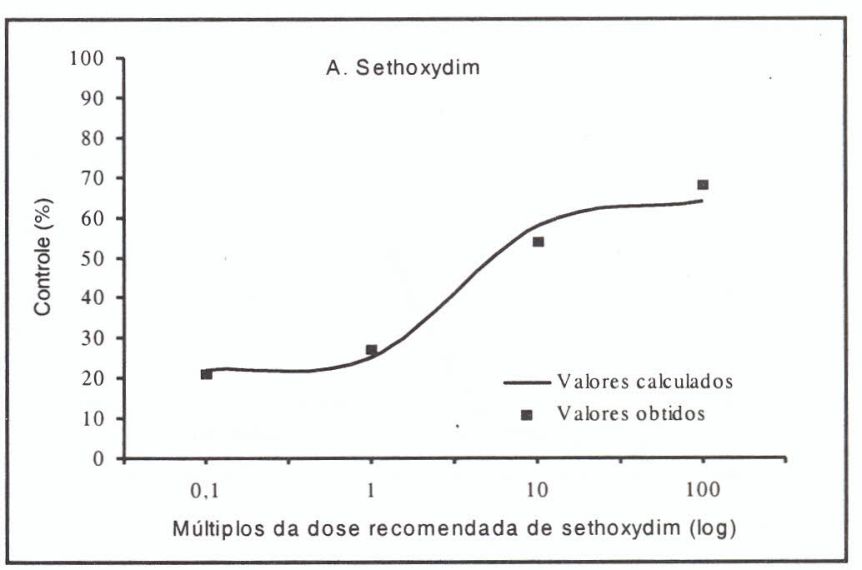

Figura 1. Curva de dose-resposta do herbicida do biótipo resistente de capim-marmelada (Brachiaria plantaginea) ao herbicida sethoxydim, aos 21 $\mathrm{DAA}$, indicando as doses de $\mathrm{GR}_{50}$ e $\mathrm{GR}_{80}$. 


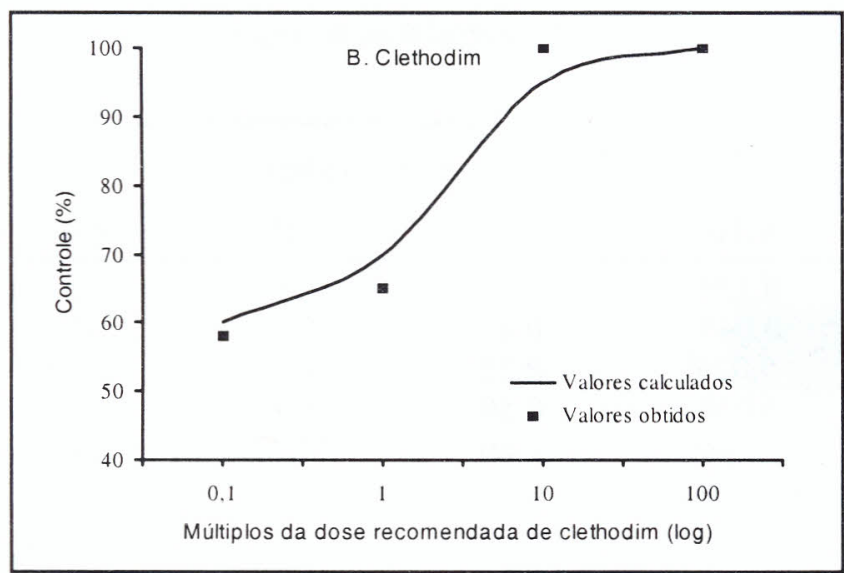

Figura 2. Curva de dose-resposta do biótipo resistente de capim-marmelada (Brachiaria plantaginea) ao herbicida clethodim, aos 21 DAA, indicando o $\mathrm{GR}_{50}$ e $\mathrm{GR}_{80}$.

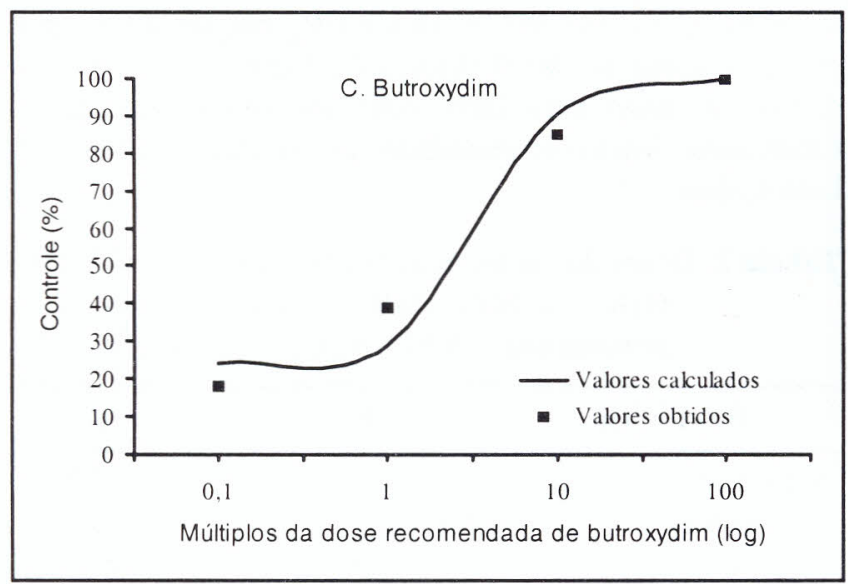

Figura 3. Curva de dose-resposta do biótipo resistente de capim-marmelada (Brachiaria plantaginea) ao herbicida butroxydim, aos 21 DAA, indicando

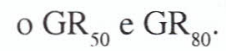

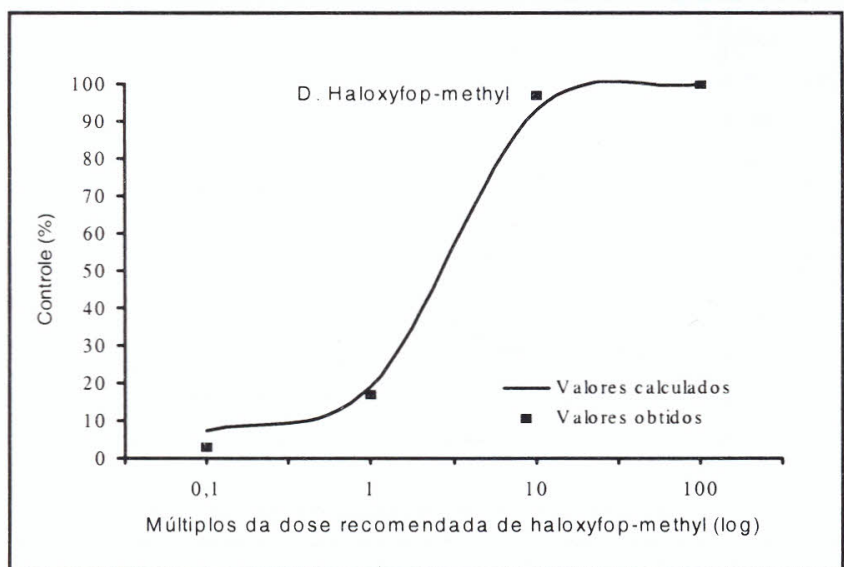

Figura 4. Curva de dose-resposta do biótipo resistente de capim-marmelada (Brachiaria plantaginea) ao herbicida haloxyfop-methyl, aos 21 DAA, indicando o $\mathrm{GR}_{50}$ e $\mathrm{GR}_{80}$.

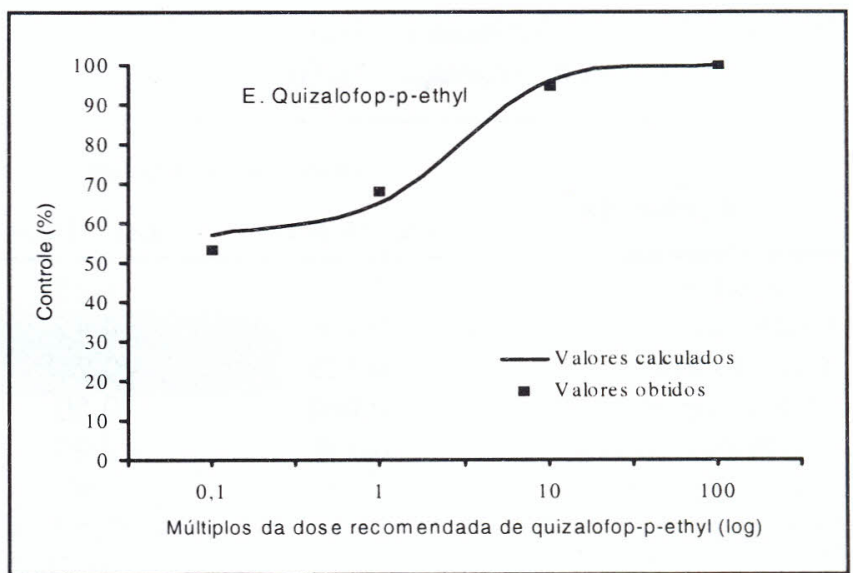

Figura 5. Curva de dose-resposta do biótipo resistente de capim-marmelada (Brachiaria plantaginea) ao herbicida quizalofop-p-ethyl, aos 21 DAA, indicando o $\mathrm{GR}_{50}$ e $\mathrm{GR}_{80}$.

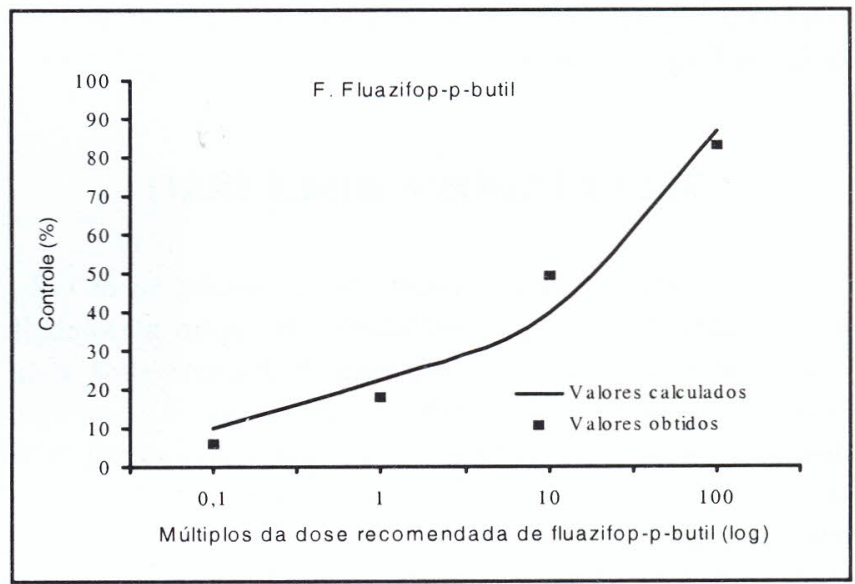

Figura 6. Curva de dose-resposta do biótipo resistente de capim-marmelada (Brachiaria plantaginea) ao herbicida fluazifop-p-butil, aos 21 DAA, indicando o $\mathrm{GR}_{50}$ e $\mathrm{GR}_{80}$.

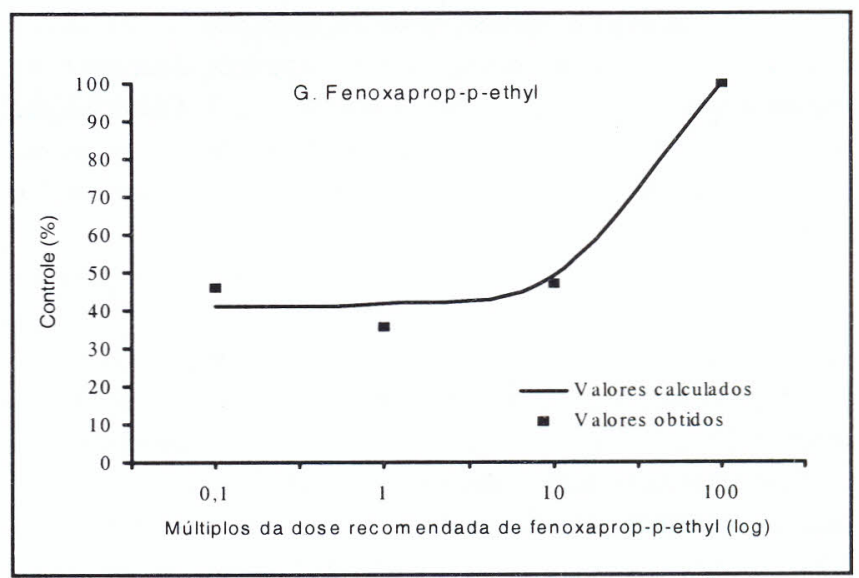

Figura 7. Curva de dose-resposta do biótipo resistente de capim-marmelada (Brachiaria plantaginea) ao herbicida fenoxaprop-p-ethyl, aos 21 DAA, indicando o $\mathrm{GR}_{50}$ e $\mathrm{GR}_{80}$.

Revista Brasileira de Herbicidas, v.2, n.3, 2001. 


\section{CONCLUSÕES}

O biótipo de capim-marmelada (Brachiaria plantaginea) estudado apresenta resistência comprovada aos herbicidas inibidores da ACCase.

Existe resistência cruzada do capim-marmelada (Brachiaria plantaginea) para os herbicidas pertencentes aos grupos químicos dos ácidos ariloxifenoxipropiônicos e das ciclohexanodionas.

O grau de resistência exibido pelo biótipo de capimmarmelada ( $B$. plantaginea) é variável dentre os herbicidas estudados, sendo que o biótipo exibiu maior grau de resistência aos herbicidas sethoxydim, fluazifop-p-butil e fenoxapropp-ethyl e, menor, aos herbicidas quizalofop-p-ethyl, clethodim, haloxyfop-methyl e butroxydim.

Os valores das doses dos índices $\mathrm{GR}_{50}$ e $\mathrm{GR}_{80}$ permitem a observação das diferenças de suscetibilidade do biótipo de capim-marmelada ( $B$. plantaginea) aos herbicidas.

\section{LITERATURA CITADA}

CHRISTOFFOLETI, P.J.; CORTEZ, M.G.; VICTORIA FILHO, R. Resistance of alexanderweed (Brachiaria plantaginea) to ACCase inhibitor herbicides in soybean from Paraná State, Brazil. In: MEETING OF THE WEED SCIENCE SOCIETY OF AMERICA. Chicago, 1998. Abstract... Chicago: WSSA, 1998. p. 65.

CORTEZ, M.G. Resistência de biótipos de Brachiaria plantaginea (Link) Hitchc. a herbicidas inibidores da acetil coenzima A carboxilase. Piracicaba: Escola Superior de Agricultura "Luiz de Queiroz" - Universidade de São Paulo, 2000. 214 p. (Tese de doutorado).

DEVINE, M.D. Mechanisms of resistance to acetyl coenzyme A carboxylase inhibitors: a review. Pesticide Science, v. 51, p. 259-264, 1997.

GAZZIERO, D.L.P.; CHRISTOFFOLETI, P.J.; MACIEL, C.D.M.; SCARAMUZA JÚNIOR, J.R. Resistência de biótipos de Brachiaria plantaginea aos herbicidas inibidores da ACCase aplicados em soja. In:
CONGRESSO BRASILEIRO DA CIÊNCIA DAS PLANTAS DANINHAS, 21. Caxambu, 1997 Resumos... Caxambú: SBCPD, 1997. p. 88.

HEAP, I. M.; KNIGHT, R. A population of ryegrass tolerant to the herbicide diclofop-methyl. Journal Austral. Ins. Agric. Sci., v. 48, p. 156-157, 1982.

HEAP, I.M.; KNIGHT, R. Variation in cross-resistance among populations of annual ryegrass (Lolium rigidum) resistant to diclofop-methyl. Australian Journal of Agricultural Research, v. 41, p. 121-128, 1990.

HEAP, I.M.; MURRAY, B.G.; LOEPPKY, H.A.; MORRISON, I.N. Resistance to aryloxyphenoxypropionate and cyclohexanodione herbicides in wild oats (Avena fatua). Weed Science, v. 41, p. 323-238, 1993.

PRESTON, C. Resistance photosystem I disrupting herbicides. In: POWLES, S. B.; HOLTUM, J. A. M., eds. Herbicide resistance in plants. Boca Raton, FL: Lewis Publishers, 1994. p. 61-82.

SAARI, J.C.; COTTERMAN, J.C.; PREMIANI, M.M. Mechanism of sulfonylurea herbicide resistance in the broad leaf weed Kochia scoparia. Plant Physiology, v.93, p.55, 1992.

SASAKI, Y.; KONISHI, T.; NAGANO, Y. The compartamentation of acetyl coenzyme A carboxylase in plants. Plant Physiology, v. 108, p. 445-449, 1995.

SELDULSKY, T. Braquiária: taxonomy of cultivated and native species in Brazil. Hoehnea, v.7, p. 99-139, 1978.

THAI, K.M.; JANA, S.; NAYLOR, J.M. Variability for response to herbicides in wild oats (Avena fatua) populations. Weed Science, v. 33, p. 829-835, 1985.

VIDAL, R.A.; FLECK, N.G. Three weed species with confirmed resistance to herbicides in Brazil. In: MEETING OF THE WEED SCIENCE SOCIETY OF AMERICA, 1997. Abstract... WSSA, 1997. p. 100. 
Revista Brasileira de Herbicidas, v.2, n.3, 2001. 